\title{
A Tactile Compass for Eyes-Free Pedestrian Navigation
}

\author{
Martin Pielot ${ }^{1}$, Benjamin Poppinga ${ }^{1}$, Wilko Heuten ${ }^{1}$, and Susanne Boll ${ }^{2}$ \\ ${ }^{1}$ OFFIS Institute for Information Technology, \\ Escherweg 2, 26121, Oldenburg, Germany \\ \{martin.pielot, benjamin, poppinga, wilko. heuten\} @offis.de \\ ${ }^{2}$ University of Oldenburg, Escherweg 2, 26121, \\ Oldenburg, Germany \\ susanne.boll@informatik.uni-oldenburg.de
}

\begin{abstract}
This paper reports from the first systematic investigation on how to guide people to a destination using the haptic feedback of a mobile phone and its experimental evaluation. The aim was to find a navigation aid that works hands-free, reduces the users' distraction, and can be realised with widely available handheld devices. To explore the design space we developed and tested different prototypes. Drawing on the results of these tests we present the concept of a tactile compass, which encodes the direction of a location "as the crow flies" in rhythmic patterns and its distance in the pause between two patterns. This paper also reports from the first experimental comparison of such tactile displays with visual navigation systems. The tactile compass was used to continuously display the location of a destination from the user's perspective (e.g. ahead, close). In a field experiment including the tactile compass and an interactive map three conditions were investigated: tactile only, visual only, and combined. The results provide evidence that cueing spatial locations in vibration patterns can form an effective and efficient navigation aid. Between the conditions, no significant differences in the navigation performance were found. The tactile compass used alone could significantly reduce the amount of distractive interaction and together with the map it improved the participants' confidence in the navigation system.
\end{abstract}

Keywords: We Mobile Accessibility, Multi-Modal Interface, Novel User Interfaces and Interaction Techniques.

\section{Introduction}

"Navigation means solving a spatial problem, usually to get from one location to one or more other locations" [17] and being spatially oriented is a key prerequisite for solving such spatial problems. Interactive maps, such as Google Maps on the iPhone, are a powerful way to provide spatial orientation when navigating in larger environments, e.g. a forest or a national park. By obtaining the user's GPS location and highlighting it on a map it becomes easy to stay oriented even for inexperienced travellers.

One challenge of using such maps is the interaction with the handheld device on the move, which may cause serious distraction from the environment. 


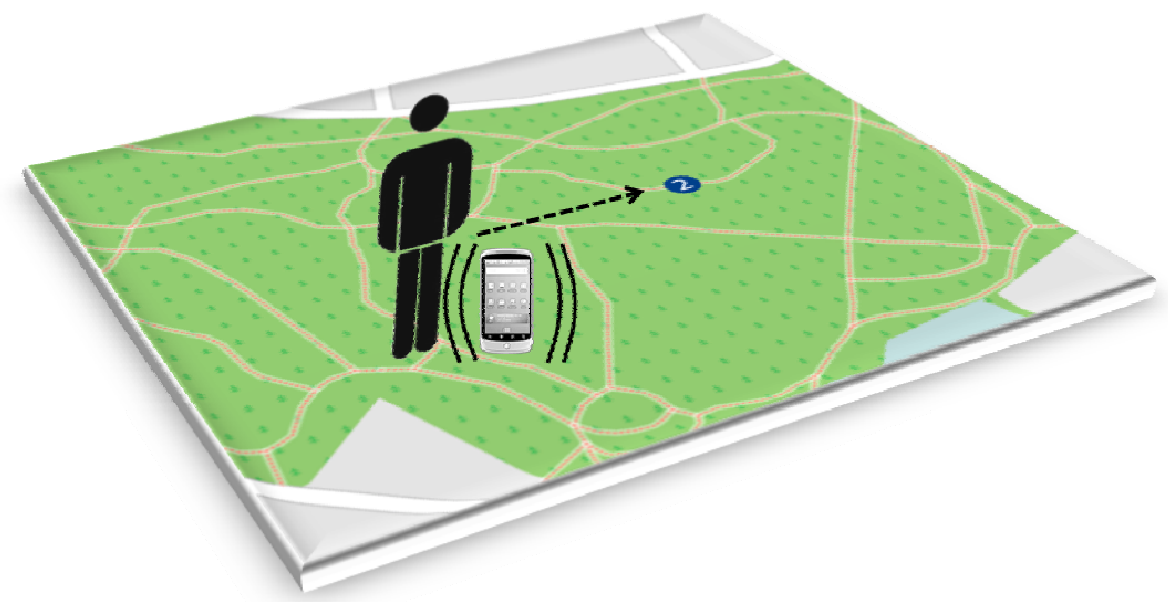

Fig. 1. A "tactile compass" encodes in which direction and how far a place is located from the perspective of the user by vibration patterns generated with a common mobile phone. Unlike wide-spread turn-by-turn wayfinding systems, this tactile compass allows a more traditional sense of navigation, empowering people to revive their inherent navigation skills.

According to a study by Madden and Rainie [11] one in six adults report to have physically bumped into another person because they were distracted using their phone. The "iPod Zombie Trance", i.e. listening to loud audio content via headphone, may result into a dangerous loss of situational awareness. Australian authorities believe that this loss is responsible for the still increasing number of pedestrian fatalities ${ }^{1}$. Beyond safety considerations, having to repeatedly looking at a tiny display to stay oriented is a stark contrast to the experience of a good hike. Auditory displays may be overheard when there is too much noise, or they may interrupt other actions, such as enjoying the hike or a chatting with a co-traveller.

To support navigation and orientation in a less distracting and obtrusive way, the sense of touch has been studied as a communication medium. It has been shown that vibro-tactile stimuli around the torso can easily be interpreted as pointing directions [27]. This kind of information presentation technique has successfully been used to "drag" people towards waypoints [28] while reducing the distraction at the same time $[3,14]$. With respect to map-based navigation it has been shown that cueing the general direction of the destination can improve the navigation performance and reduce the distraction $[15,22]$. Supporting map-based navigation is desirable, since turn-by-turn instructions may disengage users from the environment [9], lead to a worse understanding of the environments spatial layout [1], and are not necessarily more effective than maps [7].

However, the majority of the above approaches require special hardware (e.g. [28, $3,14])$ and are thus hard not widely available. Other approaches or they require proactive interaction $[13,19]$ to obtain the spatial information, which may be undesired

1 http: //www.smh.com.au/digital-life/mp3s/pedestrian-death-riseblamed-on-ipods-20100905-14w4d.html 
be the user at times. In this paper we therefore propose and investigate the concept of a tactile compass, which cues spatial information by the sense of touch and neither requires neither special hardware nor explicit interaction.

With the help of focus groups and qualitative studies we developed a set of vibration patterns that indicates the general direction and distance of a geospatial location. Similar to a traditional compass needle pointing northbound, it can be used as a tactile compass to point towards any geographical reference point, such as the travel destination (see Fig. 1). This paper reports from a systematic investigation in how to convey geospatial locations with a handheld device and our resulting tactile compass. Further, it reports from the first experimental evaluation of such tactile user interfaces comparing it with a map-based navigation system. We provide evidence that the tactile cues can reduce the distraction by decreasing the amount of interaction with the device.

\section{Related Work}

The Surveying the previous work in the field of pedestrian navigation Tscheligi and Sefelin [25] argue that landmark-based wayfinding and the consideration of the context of use are two major prerequisites for the success of pedestrian navigation systems. To address the context of use using non-visual modalities for providing spatial cues has been studied for many years. Holland et al. [6] proposed AudioGPS, where the general direction of a destination is encoded in 3D spatial audio delivered by headphones. In Strachan et al.'s GPS tune [23] a similar approach was taken. Directions are encoded in the panning of an audio track. Both groups did user trials which showed that such interfaces are effective.

If users do not want to impair their auditory perception, e.g. to avoid the loss of situation awareness, the sense of touch offers a viable alternative. Using tactile displays to deliver spatial information has been proposed more than a decade ago [24]. A popular example of such tactile displays come in the form of waist belts, such as Tsukada et al.'s [26] ActiveBelt. Eight vibro-tactile actuators are sewn into the fabrics of a waist belt. If it is worn, tactile stimuli can be produced all around the wearer's torso. As reported by van Erp et al. [27] those stimuli can intuitively be interpreted as horizontal directions. For example, a vibration near the navel is perceived as pointing forward. This type of spatial information presentation has successfully been used to facilitate waypoint navigation [28] or to cue the location of several objects $[10,4,16]$. It could be shown the even the complex visualisation of the location of the team mates in a computer game can be effectively processed despite demanding foreground tasks and lead to a significantly increased situation awareness [16]. It also could be shown that cueing the general direction of a travel destination can improve map-based navigation in virtual environments [22] and the real-world [15].

Recently, researchers have started to investigate to use the pager motors available in common mobile phones to convey spatial information with devices that are more widespread than tactile waist belts. An emerging interaction technique that can be realised with simple audio or tactile feedback is "scanning" the environment via pointing gestures. Recent examples are SoundCrumbs [12] in combination with an auditory feedback and Sweep-Shake [18] in combination with tactile feedback. The user points the device into the supposed direction of e.g. a landmark and receives 
auditory or tactile feedback when pointing into the right direction. By pointing into different directions users can scan for the landmark. Several studies have shown that people can reach given places with this interaction technique [13, 19, 29].

Both, tactile waist belts and scanning with tactile feedback have shown to be successful to support navigational tasks, but both have their drawbacks. Tactile waist belts are very intuitive but hardly available. Even if a person owned such a display it would be unlikely that it is carried along all the time. The scanning technique, on the other hand, requires nothing but a mobile phone with a built-in magnetometer. However, the need to actively point the device to probe for the spatial direction may be tedious over time.

What is still missing is a method to convey spatial directions (similar to the above described tactile belts) with a single tactile actuator, so that the tactile display (e.g. as part of the mobile phone) requires no active interaction. The question is if such a display can be realised in a way that is easy enough to effectively be used without extensive training.

\section{Design of the Tactile Compass}

In this section we describe our design that explores how to convey geospatial locations with a single vibration motor and without required explicit interaction. To underpin our design decisions we first elaborate the requirements and constraints given by the pedestrian navigation task and the device limitations. We then describe how we investigated the design space to find a set of possible solutions and illustrate the prototypes we built. We then present the results from a qualitative outdoor evaluation testing these prototypes. Drawing on our findings we illustrate the final design of our tactile compass.

\subsection{Requirements and Constraints}

Use of a Tactile Display. As mentioned in the introduction, interacting with mobile devices may be seriously distracting the user. According to a study by Madden and Rainie [11] one in six cell-owing adults report that they have physically bumped into another person because they were distracted by talking or texting on their phone. At the same time, studies showed that tactile displays used to conveying navigational information can reduce this kind of distraction significantly [3, 14]. Thus, the location of the destination to be displayed by the tactile compass should be conveyed by the sense of touch.

Support Navigation by Survey Knowledge. In modern, commercial wayfinding systems routes are typically organised as a set of waypoints. The geometric location of the waypoints in conjunction with an underlying street network can be used to generate turning instructions, such as "now turn left". However, this form of navigation guidance has shown to disengage users from the environment [9] and make people feel "bossed around" [14]. In addition, it does not take into account that pedestrians are often not as confined to the street network as cars are, e.g. when they are on a hike or freely exploring a city centre. With the design of the tactile compass we therefore aim at supporting navigation in its traditional sense. Instead of giving travellers the fastest or shortest route 
to a destination within a confined street network, the tactile compass should support travellers by indicating the direction of a geographical location "as the crow flies". Thus, it supports classic survey-based knowledge, where the traveller uses a mental map formed of a gestalt-like network of the relative locations of landmarks [8]. As spatial information always describes the relation between two objects, the location shall be described in relation to the user. This egocentric cueing is most easy to interpret and does not require considering any further reference points.

Encode Spatial Information in the Tactile Stimulus Only. The pointing-interaction proposed in previous work $[13,19,29]$ would already propose a viable solution to the above requirements. However, pointing means that the user has to interact explicitly with the mobile device to find the location of the destination. As reported in [19] users may desire constant tactile feedback but are not willing to constantly hold the device in the hand. Thus, the tactile compass should work hands-free. If no explicit interaction, such as pointing, shall be required the spatial information has to be encoded by the tactile stimulus only. While the visual and acoustic feedback can provide a huge variety of information, tactile information presentation is limited in multiple ways. On the one hand, humans can only perceive vibro-tactile stimuli if the actuator can stimulate the skin. On the other hand, the tactile feedback can only be varied in limited ways, depending on the used actuator technology. Most mobile phones only allow turning the electric motor on and off, which generates vibration through an off-centred weight attached to the motor axis. Thus, only vibrations with different length or rhythms can be created. Other parameters (see [2]), such as the frequency, the amplitude, or the waveform of the vibration cannot be altered.

\subsection{Investigation of the Design Space}

In order to investigate how to cue the location of a place in relation to the user's location we conducted a focus group with five colleagues from our research group. We brainstormed potential solutions, identified common concepts, and derived five prototypes.

The most prominent aspect we identified was that every method we could think of either represented direction or distance information. Examples of this are describing locations by their distance and their azimuth with respect to a person using the clock face, such as "2 o'clock, 150 metres". All other approaches would require external means of geospatial referencing, such as landmarks or GPS coordinates. The other prominent aspect was whether the presentation was binary or if it had multiple levels. For example, being near the destination versus being not near the destination would be a binary distance representation. The distance to a destination in metres would be an n-ary presentation. While some of these combinations are reasonably suited to the problem, others are obviously insufficient to guide a pedestrian efficiently to a destination. Indicating in a binary way whether the user is at the destination or not would not be much help in reaching the waypoint in the first place.

\subsection{Implementation of Prototype Methods}

In summary we assumed that conveying absolute distances, distance changes over time, and general directions were the most promising ways of guiding a person to a 
place or destination. We used these elements of our design space to construct five different methods to guide people to a given geo location.

Technically, each method allows specifying a destination by a pair of latitude/longitude coordinates. Further, each method is fed with the GPS signal of the mobile phone, so it knows the user's geo-location, heading and walking speed. For each method, we had to design a mapping from the information that shall be presented to a set of rhythm patterns. Further, we already optimised the methods were it seemed suitable although that may have diluted the "pure" aspects.

Approaching/Departing. This method focuses on conveying the relative distance to the location as it changes over time. It indicates whether the user is walking towards presented location or not. User can reach the destination following the "walking towards the location" signal. Walking towards the waypoint is encoded in a single long $(240 \mathrm{~ms})$ pulse. Not walking towards the location is encoded by two short pulses of $120 \mathrm{~ms}$ with a pause of $120 \mathrm{~ms}$ between them. We defined "approaching" when the location is within a cone of $2 \times 60^{\circ}$ in front of the user.

Hot'n'Cold. This method draws on the child game hot 'n' cold. It indicates the absolute distance to the location. By moving into the direction where the signal gets "hotter" the user will eventually reach the destination. Therefore the method continuously generates a single tactile pulse of $120 \mathrm{~ms}$. The distance between the user and the location to each is encoded in the pause between the pulses. The closer the user gets to the waypoint the shorter the pause becomes. The pause durations range from $5000 \mathrm{~ms}$ (1000 metres or beyond) to $300 \mathrm{~ms}$ (reached the destination).

Left, Right, Ahead. This method encodes if the waypoint is left, right, or ahead of the user. The waypoint being left of the user is encoded in two pulses of $120 \mathrm{~ms}$. The waypoint being right of the user is encoded in three pulses of $120 \mathrm{~ms}$. To avoid having the user running zigzag we introduced a small frontal corridor, which indicates if the location is in a cone of $30^{\circ}$ in front of the user. This case is encoded by a single $120 \mathrm{~ms}$ pulse.

Continuous Direction. This method encodes the exact direction of the location in the $360^{\circ}$ full circle. It therefore creates a rhythm pattern that is altered continuously depending on the direction to present. If users are able to interpret the rhythm patterns they can just "read" the direction of the location and head into the respective way. The basic principle of the method is to encode the direction in the relative lengths of two vibration pulses. If the waypoint was dead ahead both pulses have a length of $80 \mathrm{~ms}$. If the location is right of the user, the length of the second pulse is increased. The further right the location gets the longer the second pulse becomes. Locations to the left of the user are displayed by increasing the length of the first pulse.

During our repeated early tries, we found that it was necessary to communicate when a waypoint is being reached and when the GPS signal is becoming too bad. Thus, each method implemented two basic Tactons: A short reoccurring pulse followed by a long pause indicates insufficient GPS signal quality. A sequence of three pulses (short, long, short) was used to announce that the destination has been reached: the first and the last pulse had a duration of $80 \mathrm{~ms}$, while the middle pulse had a duration of $500 \mathrm{~ms}$. The pause between those pulses was $120 \mathrm{~ms}$. 


\subsection{Qualitative Evaluation of Designs}

We conducted a pilot study to figure out, which of the design approaches is most suited as a tactile compass for navigation. Therefore, the four methods were prototypically implemented on a Nokia N95. In addition, the prototype allowed us to define and store geo locations. One then could select a geo location and choose a method to display its location.

Method. The pilot study was conducted in a residential area near the OFFIS Institute for Information Technology in Oldenburg, Germany. The geo coordinates of four places were designated as destinations and stored in the prototype. Seven participants, who were partially familiar with the covered area, took part in the study. Each participant had to reach all destinations in the same order. For every destination another method was used. To avoid order effects the order of methods was randomised amongst the participants to avoid sequence effects. Beyond the tactile feedback the participants had no other cues about the location of the destination.

Qualitative data was collected through thinking aloud as well as video recording. All participants signed an informed consent prior to the study. At the end of the study the participants were asked to rank the design approaches and discuss their impressions about each of them.

Results and Discussion. In all but four occasions the participants were able to reach the given destination. In general, all methods were found to be reasonably effective. The four breakdowns were instances of a participant standing on one side of a building while the destination was located on the other side.

Many participants did not like the distance-based methods since they required them to actively seek the destination. Direction-based methods were preferred over distancebased methods. Five informants named the Continuous Direction method as the most preferred one. Although it was found to be the most complex method, the informants appreciated its rich feedback. In particular, informants appreciated that they could observe how the direction of the destination slowly changes while they are moving.

In some cases obstacles such as larger buildings or blind alleys with the destination point behind them lead to confusion. In order to avoid the obstacles, the participants had to change their direction and accept the advice that their direction is wrong until they passed the obstacle. This caused irritations because the participants were not happy with foregoing the instructions of the system. It was stated that more waypoints on the route could help to resolve this issue.

However, there are a few limitations to the results. The methods we tested are only instances of the design space we laid out above. Neither the design space is necessarily complete nor might the instances have been the best examples for the entities of the design space. Nevertheless, the results suggest that participants prefer direction-based methods with rich information and are willing to accept complexity. Thus, one challenge would be how to encode as much information as possible in the tactile cues, before they become too complex to be understood.

\subsection{The Tactile Compass' Design}

Drawing on the results from the above qualitative evaluation of the design methods we built a method that combined the "best of all worlds". We based it on the 
Continuous Direction method, as this method was preferred by the users. As seen in Figure 2 we advanced the method by indicating locations behind the user in three short pulses. In addition, we added an absolute distance cue similar to the one we used in the Hot 'n' Cold method. The method therefore alters the pause between the patterns. The closer the user gets to the destination the shorter the pause becomes (see Figure 3). To improve the ability to discriminate the pulses we increase the length of the short pulses and the pauses between the single pulses of a pattern to $120 \mathrm{~ms}$.

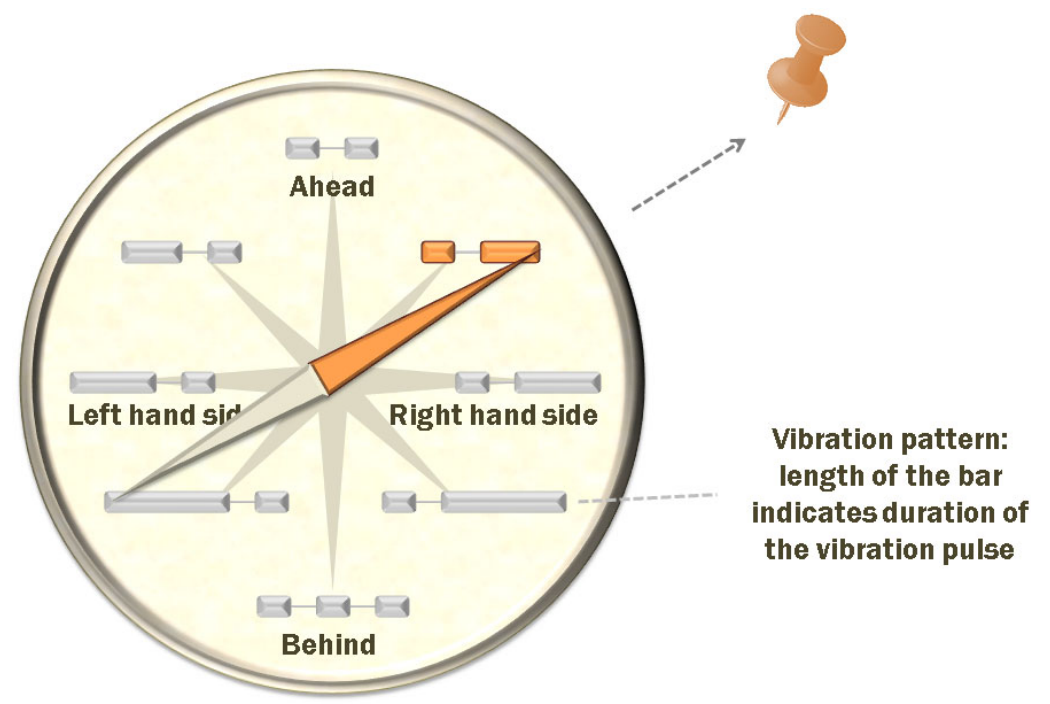

Fig. 2. The pointing direction is encoded in the relative length of two pulses. In this illustration the geographic reference point is somewhat to the right-hand side, so the first pulse remains short while the length of the second pulse is slightly increased.

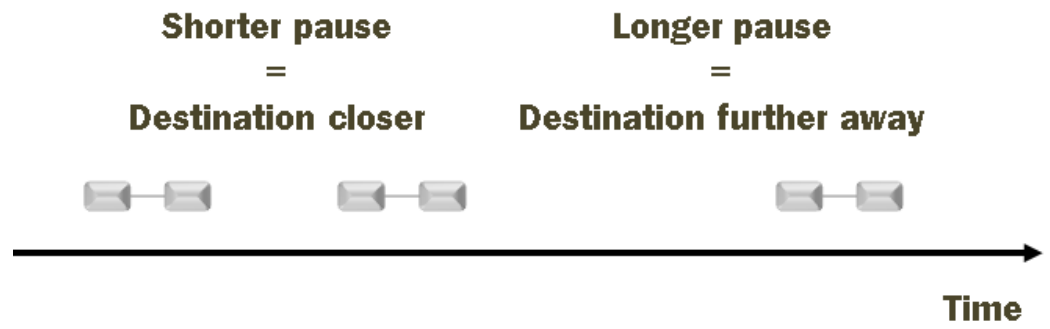

Fig. 3. The distance is encoded in the pause between a set of pulses. The shorter the pause becomes the closer the user is to the presented location.

\section{Field Study}

The tactile compass was evaluated as a navigation aid in a field study. The study took place in a city forest. Fourteen participants had to reach a given destination, either by a 
map, by the tactile compass, or both navigation aids. The goals where it investigate (1) if the tactile compass can effectively guide pedestrians to a given location, (2) how well it can keep up with a map, and (3) if the tactile cues have a positive effect on the participants distraction.

\subsection{Material}

For the baseline we had to choose between a turn-by-turn navigation system and a map. We decided for the map for two reasons: first, maps are still used often and heavily relied on, in particular in an exploration scenario. Second, Ishikawa et al. [7, 20] suggest that maps are still superior to navigation systems in terms of navigation performance. To provide the map we used a custom built application that displays the user's position and walking direction on a map layer using OpenStreetMap data.

The evaluation took place in a city forest. The area offers lots of winding paths and combines dense forest with a couple of open meadows that could be used as shortcuts. Additionally, the forest contains lots of landmarks that we used to measure how attentive the participants could remain with respect to the environment.

For the evaluation we defined three places (see Figure 4). The application could be configured to show one of those places on the map and display its location through the tactile compass. No route was displayed on the map and no turning instructions were given by the application. Thus, the participants had to find their own way to the given destination.

The places were arranged in a way that there was never a direct path or line of sight between the start and the place to reach. The route always included detours (unless the participants walked cross-country). Thus, the task of reaching the destination was never trivial for the participants.
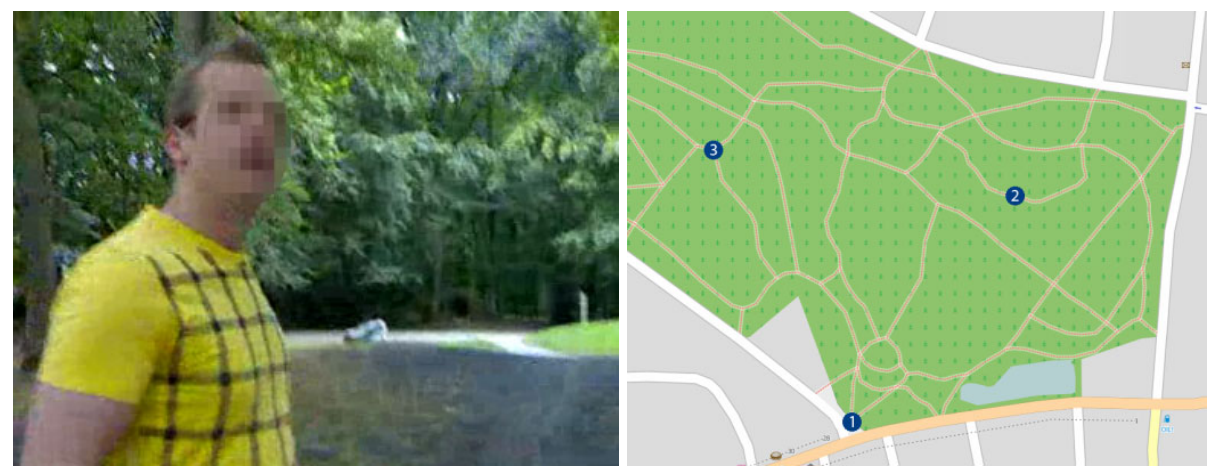

Fig. 4. Left: the city forest that we used as evaluation environment: the dots mark the three places we used as destination in the navigation tasks. Right: a participant during the field study while learning the tactile compass' vibration patterns.

\subsection{Participants}

Fourteen participants (four female) took part in the study. Their age ranged from 14 to 53 with an average of 28.25 (SD 11.51). According to the Santa Barbara 
Sense-of-Direction Scale (SBSOD) [5] they reported an average sense-of-direction (3.07, SD 1.14, with possible scores ranging from 1 to 5). All of the participants had little or no knowledge about the spatial layout of the evaluation environment. Since the evaluation was video-recorded the participants signed an informed consent. No payment was provided for the participation.

\subsection{Design}

The independent variable was the type of the navigation aid. To isolate effects caused by the map and by the tactile cues, we used three levels: \{map, tactile, and map \& tactile\}. Map denoted the condition where the participants only used the map displayed on the mobile device's screen. In the tactile condition the device only presented direction and distance of the destination via a tactile compass as described in Section 3.5. The map \& tactile condition combined the use of the visual map and the tactile compass. We used a within-subjects design with all participants using all three navigation aids in random order. The following measures were taken in order to assess the navigation performance and distraction in each condition:

Navigation Performance. Similar to what has been reported in previous studies [21, $20,15]$ on evaluations of navigation systems we measured navigation performance in terms of completion time and occurrences of disorientation events. The time the participants needed to reach the destination from the starting point of each condition was considered as completion time. Disorientation events were defined as situations where the participants stopped for more than 10 seconds, or for 5 seconds when they expressed their disorientation verbally. In addition the participants were asked to rate on a five-point Likert scale how confident they were with their navigation decisions. We did not measure navigation errors, since there was no "correct" route, and thus the concept of a navigation error made no sense.

Distraction. For measuring distraction we combined three measures. First, the participants were asked to count the number of benches they saw during the study. From the number of the detected and the missed benches we then computed a detection rate. Second, the participants rated how much they felt distracted by the device on a five-point Likert-scale. Third, we measured how long the participants visibly interacted with the device. Visible interaction was defined as any situation with where a participant interacts with the mobile device in a way that was visible to the experimenter. Even a mere glance at the display was considered as interaction as long as it was clearly perceivable by the experimenter. We divided the cumulated time of visible interaction by the completion time to get a time ratio for each condition.

\subsection{Procedure}

Prior to the study, informed consents were sent out to the potential participants. Only those participants who signed the consent forms were invited to the study. Initially, the experimenters re-explained the tasks (navigate to the given location using any path they want). The participants were introduced to the three conditions and allowed to train them. 
Prior to the first session, the participants were informed that they had to count all the benches they could find, and report the number once they had reached the destination. The places shown in Figure 4 had to be reached in the given order $(1,2$, and 3). During the navigation task one experimenter followed the participant in some distance and recorded their action with a video camera.

Once the participants had reached a destination the completion time was noted. The participants were asked to report the number of detected benches and rate the subjective level of confidence about the navigation decisions and the subjective level of distraction for the current navigation aid. Afterwards, the condition was switched and the next place was selected as destination, until all three places had been reached. The whole procedure took about 45 minutes for each participant.

\section{Results}

All participants reached the destinations in all trials within a reasonable amount of time. No notable performance breakdown was observed in any of the conditions. In the following we report our quantitative findings as well as participant comments and our observations.

\subsection{Quantitative Results}

The quantitative results were extracted from the questionnaires and the video recordings. Table 1 shows the mean results for every dependent variable grouped by the condition. Statistical significance was analysed using ANOVA and Tukey posthoc tests for the ratio variables and the Friedman Test and Benferroni corrected Wilcoxon Signed Rank tests for the questionnaire's Likert scale results.

Table 1. Quantitative average results by condition. The subjective measures are the results of five-point Likert-scales, where 5 means highest. Bold faced numbers indicate that a significant effect on its condition was found.

\begin{tabular}{|c|c|c|c|}
\hline Measure/Condition & map & tactile & map \& $t$ \\
\hline \multicolumn{4}{|l|}{ Navigation Performance } \\
\hline Completion time (s) & 361.6 & 464.3 & 398.6 \\
\hline Disorientation ev. (\#) & 0.21 & 0.50 & 0.29 \\
\hline Confidence * & 4 & 4 & 5 \\
\hline \multicolumn{4}{|l|}{ Distraction } \\
\hline Interaction time $(\%) * * *$ & 35.75 & 6.41 & 27.82 \\
\hline Benches discovered (\%) & 46.36 & 50.41 & 41.26 \\
\hline Subj. Distraction & 3 & 3 & 3 \\
\hline
\end{tabular}

Navigation Performance. There was a significant effect of the navigation aid on the subjective confidence of the participants $\left(\mathrm{X}^{2}(2)=8.45, \mathrm{p}<.05\right)$. It was significantly higher when both navigation aids were used in combination (both $p<.01$ ). The 
difference between map and tactile was not statistically significant. Otherwise, no significant effects on the navigation performance were found. Neither the completion time $\left(\mathrm{F}_{2}=1.08, \mathrm{p}=.35\right)$ nor the number of disorientation events $\left(\mathrm{F}_{2}=.58, \mathrm{p}=.56\right)$ differed significantly between the conditions.

Distraction. There was a significant effect on the interaction time $\left(F_{2}=15.26, p<\right.$ .001). A Tukey HSD post-hoc test showed that the participants interacted significantly less often with the device in the tactile condition compared to the map condition $(p<.001)$ and the map \& tactile condition $(p<.001)$. The difference between map and map \& tactile was not significant. With respect of the number of benches found, no significant effect could be observed $\left(\mathrm{F}_{2}=.48, \mathrm{p}=.62\right)$. Similarly, the difference in the subjective distraction was not statistically significant $\left(\mathrm{X}^{2}(2)=.894, \mathrm{p}=.64\right)$.

\subsection{Comments and Observations}

Visible Interaction. It did not bring any value to the participants, but still we found instance of visible interaction in the tactile condition. Examples for visible interaction where for examples participants unlocking the screen saver (although there was nothing to see), playing with the phone's slider, or simply looking at the display.

Cross Country Walking. Most participants stuck to the given paths. This is surprising as the city forest contains many open areas and people are allowed to enter them. Only a few participants walked cross country. This always involved the tactile compass and mostly happened in the tactile condition. These participants also "scored" the fastest overall completion times.

Training Effect. Since all participants used the tactile compass two times, in the tactile condition and in the map \& tactile condition, we could observe the learning effect in our quantitative results. They results show that in terms of navigation performance participants often performed better with the tactile compass, when they used it for the second time. However, the differences were not statistically significant.

Overview vs. Direction Cueing. Four participants stated that they had missed having an overview of the environment in the tactile condition. They said that the map in addition gave them an impression about further waypoints on the route, which improved their confidence. Three participants said that they preferred the combination of both navigation aids, since they cancelled out each other's weaknesses and provided the richest set of information.

\section{Discussion}

All navigation aids, the tactile compass, the map, and the combination of both, allowed the participants to effectively reach the given destinations. The tactile compass could significantly reduce the time participants interacted with the handheld device. Combining both navigation aids significantly increased the participants' subjective confidence in the system.

The results show that the approach of conveying a general direction by rather unintuitive vibration patterns can form an effective navigation aid. This goes in line 
with previous findings. Several studies showed that conveying general directions are sufficient to guide a traveller to a destination [13, 18, 19]. This paper extends the previous work by reporting the first experiment comparing this navigation technique together with and against a map. Although the participants were not familiar with the tactile compass and the fact that it conveys less overview than a map, we did not find a significant disadvantage in the objective navigation performance. We carefully conclude that the tactile compass is not only effective but also reasonably efficient.

Navigation Performance. Previous studies show cueing the destination's direction by an egocentric tactile cue can improve the efficiency of navigating with a map [15, 22]. In these studies the participants were wearing a tactile waist belt pointing at the destination while navigating by a map. In both study environments, a virtual world [22] and a village [15], the navigation performance significantly improved. This study could not confirm these findings, except for the improved confidence of the participants. The two relevant differences between these and the study presented here are the environments (virtual environment and village vs. forest) and the used tactile display (tactile waist belt vs. tactile rhythm patterns). The environment of the forest might have penalised bad navigation choices less than the environments from the previous work, as it allows walking cross country and the path network is very dense. Participants making a bad route choice could often correct that only shortly after. We also assume that the tactile compass is less intuitive than the very powerful waist belts. This may have led to performance penalties which did not occur in the above studies.

However, the tactile compass also showed that it may be a highly effective navigation tool when used with some training and/or in unconventional ways. Especially in the beginning some participants had difficulties to interpret the tactile compass, which lead to a high variance in the navigation performance measures. Both, the highest (1162s) as well as the lowest (244s) completion times were measured for the tactile compass only condition. The highest completion times occurred when the participants started with the tactile compass and still had difficulties interpreting it despite the training session. This also correlated with the bulk of the disorientation events. Five out of seven disorientation events with the tactile compass occurred in only two sessions that also resulted in the two highest completion times. On the other hand, the fastest completion times were also measured in the tactile condition, when the participants had fewer difficulties in learning the tactile compass' vibration patterns. These users also showed a tendency to take shortcuts by walking cross-country.

Distraction. Previous work indicates that tactile displays used to convey navigation information can improve the users' attention. It was found that people are able to spot more entities they are tasked to search for [3] and pay more attention to their immediate surroundings, such as obstacles and other people [14]. However, in the study presented here the participants neither felt less distracted nor spotted more benches. Again, related work [3,14] studied tactile vests and waist belts, which are presumably more intuitive to interpret than the rhythm patterns of the tactile compass. We assume that the participants had to devote too much attention to the tactile cues, so the increase in cognitive workload cancelled out the advantages of the eyes-free usage. More training might reduce this disadvantage. Still, there was a positive tendency, as the participants found most benches in the tactile condition. 
The visible distraction by the device was significantly affected by the experimental manipulation. In the tactile condition the participants interacted significantly less often visibly with the mobile device. At the first glance, this result might seem obvious, as there was no map to look at in the tactile condition. However, visible interaction was also possible in the tactile condition. Examples are the participants visibly listening to the tactile patterns or holding the device by the ear to "hear" the patterns. Further, the interaction with the map could have been far less, so the differences would be been insignificant. In fact, the frequent visible interaction with the map (about 28-36\% in the conditions where the map was available) indicates how dangerously distracting a map can be. These findings go in line with previous studies [15].

Limitations. As every experiment, one limitation of the findings is that they are subject to Hume's problem of induction. A single experiment cannot prove that the findings will be reproduced in different settings. This means, we do not know how the tactile compass would perform in crowded Tokyo by night, a deep Finish forest in the winter, or the endless plains of the Mid West of the US.

Nevertheless, the study has shown that cueing general directions in tactile patterns - although being not necessarily intuitive - is an effective navigation aid. It showed that pedestrians can find a path to a destination in a complex, winding path network with the tactile compass only. Since most participants only used existing paths and there was mostly no line of sight between them and the destination, there is some chance that the results could be replicated in other environments with road networks as well.

\section{Conclusions}

In this paper we presented the design and evaluation of a tactile compass for everyday mobile devices that encodes the direction and distance of geographic location in vibration patterns. The contribution of the paper is twofold: (1) we provide evidence that people can effectively navigate by vibration patterns cueing the geospatial location of a destination "as the crow flies". For this form of information presentation we established the term tactile compass. (2) In addition, we could show that such tactile information cueing has a measurable positive effect on the user's attention, which may be threatened by today's display-centric user interfaces.

With the tactile compass, it becomes possible to create a "tactile sense of direction" with the phone resting in the pocket. It provides new ways to overcome the commanding nature of turn-by-turn instructions and the dangerous "head down" interaction caused by visual maps used on the move. This new "sense" allows to freely exploring the environment while remaining a sense of one's own movement in relation to a point of reference. Beyond navigation systems a tactile compass might boost any location-based service that communicates spatial information, such as a friend finder (Google Latitude) or a POI search app (AroundMe). It is associated to the vision of a more traditional understanding of navigation, more than going from $\mathrm{A}$ to B as fast as possible, rather in a sense of Kurt Tucholsky (1890-1935) stating that "Umwege erweitern die Ortskenntnis" ("Detours expand the knowledge of a place"). 
Future work needs to investigate if this "tactile sense of direction" can be made more intuitive. Intuitiveness may be achieved by revising the patterns and the implicit interaction until finding the simplest but yet sufficiently powerful variant. The simple the pattern the more beneficial the tactile compass will be in terms of distraction and cognitive workload. Further, the question of how far this concept scales has to be addressed. The presented study has shown that the tactile compass works in smaller scales of roughly a few 100 metres travel distance. We do not know how well these findings scale for longer distances (e.g. several miles) and different environments (e.g. suburbia or city centre). To support navigation at a larger scale, an approach could be to introduce intermediate landmarks to avoid walking into dead ends. However, to avoid curbing the user again, as turn-by-turn instructions do, such intermediate landmarks should be placed at reasonable intervals, so that users "hop" along a set of interesting places until reaching the destination.

Acknowledgements. The authors are grateful to the European Commission, which co-funds the IP HaptiMap (FP7-ICT-224675). We like to thank our colleagues for sharing their ideas with us.

\section{References}

[1] Aslan, I., Schwalm, M., Baus, J., Krüger, A., Schwartz, T.: Acquisition of spatial knowledge in location aware mobile pedestrian navigation systems. In: MobileHCI 2006: Proceedings of the 8th Conference on Human-Computer Interaction with Mobile Devices and Services, pp. 105-108. ACM, New York (2006)

[2] Brewster, S., Brown, L.M.: Tactons: structured tactile messages for non-visual information display. In: Proc. of the Australasian Conference on User Interface (2004)

[3] Elliott, L.R., van Erp, J., Redden, E.S., Duistermaat, M.: Field-based validation of a tactile navigation device. IEEE Transactions on Haptics 99 (2010) (preprints)

[4] Ferscha, A., Emsenhuber, B., Riener, A., Holzmann, C., Hechinger, M., Hochreiter, D., Franz, M., Zeidler, A., dos Santos Rocha, M., Klein, C.: Vibro-tactile space-awareness. In: Ubicomp, Adjunct Proceedings (2008)

[5] Hegarty, M., Richardson, A.E., Montello, D.R., Lovelace, K., Subbiah, I.: Development of a self-report measure of environmental spatial ability. Intelligence 30, 425-447 (2002)

[6] Holland, S., Morse, D.R., Gedenryd, H.: Audiogps: Spatial audio navigation with a minimal attention interface. Personal Ubiquitous Comput. 6(4), 253-259 (2002)

[7] Ishikawa, T., Fujiwara, H., Imai, O., Okabe, A.: Wayfinding with a gps-based mobile navigation system: A comparison with maps and direct experience. Journal of Environmental Psychology 28(1), 74-82 (2008)

[8] Lawton, C.A.: Gender differences in way-finding strategies: Relationship to spatial ability and spatial anxiety. Sex Roles 30(11-12), 765-779 (1994)

[9] Leshed, G., Velden, T., Rieger, O., Kot, B., Sengers, P.: In-car gps navigation: engagement with and disengagement from the environment. In: CHI 2008: Proceeding of the Twenty-Sixth Annual SIGCHI Conference on Human Factors in Computing Systems, pp. 1675-1684. ACM, New York (2008)

[10] Lindeman, R.W., Sibert, J.L., Mendez-Mendez, E., Patil, S., Phifer, D.: Effectiveness of directional vibrotactile cuing on a building-clearing task. In: $\mathrm{CHI}$ 2005: Proceedings of the SIGCHI Conference on Human Factors in Computing Systems, pp. 271-280. ACM, New York (2005) 
[11] Madden, M., Rainie, L.: Adults and cell phone distractions. Technical report. Pew Research Center (2010)

[12] Magnusson, C., Breidegard, B., Rassmus-Gruhn, K.: Soundcrumbs - hansel and gretel in the 21st century. In: HAID 2009: 4th International Workshop, Haptic and Audio Interaction Design (2009)

[13] Magnusson, C., Rassmus-Gröhn, K., Szymczak, D.: The influence of angle size in navigation applications using pointing gestures. In: Nordahl, R., Serafin, S., Fontana, F., Brewster, S. (eds.) HAID 2010. LNCS, vol. 6306, pp. 107-116. Springer, Heidelberg (2010)

[14] Pielot, M., Boll, S.: Tactile Wayfinder: comparison of tactile waypoint navigation with commercial pedestrian navigation systems. In: The Eighth International Conference on Pervasive Computing, Helsinki, Finland (2010)

[15] Pielot, M., Henze, N., Boll, S.: Supporting paper map-based navigation with tactile cues. In: MobileHCI 2009 (2009)

[16] Pielot, M., Krull, O., Boll, S.: Where is my team? supporting situation awareness with tactile displays. In: CHI 2010: Proceeding of the Twenty-Eighth Annual SIGCHI Conference on Human Factors in Computing Systems. ACM, New York (2010)

[17] Platzer, E.: Spatial Cognition Research: The Human Navigation Process and its Comparability in Complex Real and Virtual Envrionments, PhD thesis. Universit der Bundeswehr Munchen (2005)

[18] Robinson, S., Eslambolchilar, P., Jones, M.: Sweep-shake: finding digital resources in physical environments. In: MobileHCI 2009: Proceedings of the 11th International Conference on Human-Computer Interaction with Mobile Devices and Services, pp. 110. ACM, New York (2009)

[19] Robinson, S., Jones, M., Eslambolchilar, P., Murray-Smith, R., Lindborg, M.: "I Did It My Way": Moving away from the tyranny of turn-by-turn pedestrian navigation. In: MobileHCI 2010: Proceedings of the 12th International Conference on Human-Computer Interaction with Mobile Devices and Services. ACM, New York (2010)

[20] Rukzio, E., Müller, M., Hardy, R.: Design, implementation and evaluation of a novel public display for pedestrian navigation: the rotating compass. In: CHI 2009: Proceedings of the 27th International Conference on Human Factors in Computing Systems, pp. 113122. ACM, New York (2009)

[21] Seager, W., Fraser, D.S.: Comparing physical, automatic and manual map rotation for pedestrian navigation. In: CHI 2007: Proceedings of the SIGCHI Conference on Human Factors in Computing Systems, pp. 767-776. ACM, New York (2007)

[22] Smets, N.J.J.M., te Brake, G.M., Neerincx, M.A., Lindenberg, J.: Effects of mobile map orientation and tactile feedback on navigation speed and situation awareness. In: MobileHCI 2008: Proceedings of the 10th International Conference on Human Computer Interaction with Mobile Devices and Services, pp. 73-80. ACM, New York (2008)

[23] Strachan, S., Eslambolchilar, P., Murray-Smith, R., Hughes, S., O'Modhrain, S.: Gpstunes: controlling navigation via audio feedback. In: MobileHCI 2005: Proceedings of the 7th International Conference on Human Computer Interaction with Mobile Devices \& Services, pp. 275-278. ACM, New York (2005)

[24] Tan, H.Z., Pentland, A.: Tactual displays for wearable computing. In: ISWC 1997: Proceedings of the 1st IEEE International Symposium on Wearable Computers, p. 84. IEEE Computer Society, Washington, DC, USA (1997)

[25] Tscheligi, M., Sefelin, R.: Mobile navigation support for pedestrians: can it work and does it pay off? Interactions 13, 31-33 (2006) 
[26] Tsukada, K., Yasumura, M.: ActiveBelt: Belt-type wearable tactile display for directional navigation. In: Davies, N., Mynatt, E.D., Siio, I. (eds.) UbiComp 2004. LNCS, vol. 3205, pp. 384-399. Springer, Heidelberg (2004)

[27] van Erp, J.B.F.: Presenting directions with a vibrotactile torso display. Ergonomics 48, 302-313 (2005)

[28] van Erp, J.B.F., van Veen, H.A.H.C., Jansen, C., Dobbins, T.: Waypoint navigation with a vibrotactile waist belt. ACM Transactions on Applied Perception 2(2), 106-117 (2005)

[29] Williamson, J., Robinson, S., Stewart, C., Murray-Smith, R., Jones, M., Brewster, S.: Social gravity: A virtual elastic tether for casual, privacy-preserving pedestrian rendezvous. In: CHI 2010: Proceeding of the Twenty-Eighth Annual SIGCHI Conference on Human Factors in Computing Systems. ACM, New York (2010) 